\title{
SBC2011-53260
}

\section{APPLICATION OF THE THEORY OF INTERACTING CONTINUA TO BLOOD FLOW}

\author{
Mehrdad Massoudi \\ U. S. Department of Energy, National Energy \\ Technology Laboratory (NETL) \\ Pittsburgh, PA, 15236, USA
}

Samuel J. Hund

Department of Biomedical Engineering,

Carnegie Mellon University

Pittsburgh, PA, 15213, USA

\section{INTRODUCTION}

Micro-scale investigations of the flow and deformation of blood and its formed elements have been studied for many years. Early in vitro investigations in the rotational viscometers or small glass tubes revealed important rheological properties such as the reduced blood apparent viscosity, Fahraeus effect and Fahraeus-Lindqvist effect [1], exhibiting the nonhomogeneous property of blood in microcirculation. We have applied Mixture Theory, also known as Theory of Interacting Continua, to study and model this property of blood $[2,3]$. This approach holds great promise for predicting the trafficking of RBCs in micro-scale flows (such as the depletion layer near the wall), andother unique hemorheological phenomena relevant to blood trauma. The blood is assumed to be composed of an RBC component modeled as a nonlinear fluid, suspended in plasma, modeled as a linearly viscous fluid.

\section{METHODS}

\section{GOVERNING EQUATIONS}

Assuming no interconversion of mass between the two components, conservation of mass for the plasma and the RBCs take the form:

$$
\frac{\partial \rho_{1}}{\partial \mathrm{t}}+\operatorname{div}\left(\rho_{1} \mathbf{v}_{1}\right)=0, \quad \frac{\partial \rho_{2}}{\partial \mathrm{t}}+\operatorname{div}\left(\rho_{2} \mathbf{v}_{2}\right)=0
$$

where $\rho_{1}$ and $\rho_{2}$ are the bulk densities of the mixture components given by $\rho_{1}=\gamma \rho_{f}, \rho_{2}=\phi \rho_{s}$, where $\rho_{f}$ is the density of the pure plasma, $\rho_{s}$ is the density of the pure RBCs, $\gamma$ is the volume fraction of the plasma component, and $\phi$ is the volume fraction of the RBC component. For a saturated mixture, $\gamma=1-\phi$.

\author{
Jeongho Kim \\ Department of Biomedical Engineering, \\ Carnegie Mellon University \\ Pittsburgh, PA, 15213, USA \\ James F. Antaki \\ Department of Biomedical Engineering, \\ Carnegie Mellon University \\ Pittsburgh, PA, 15213, USA
}

Let $\mathbf{T}_{1}$ and $\mathbf{T}_{2}$ denote the partial stress tensors. Then, the balance of linear momentum equations for the two components is given by [4]:

$$
\begin{aligned}
& \rho_{1} \frac{D \mathbf{v}_{1}}{D t}=\operatorname{div}\left(\mathbf{T}_{1}\right)+\rho_{1} \mathbf{b}_{1}+\mathbf{f}_{\mathbf{I}} \\
& \rho_{2} \frac{D \mathbf{v}_{2}}{D t}=\operatorname{div}\left(\mathbf{T}_{2}\right)+\rho_{2} \mathbf{b}_{2}-\mathbf{f}_{\mathbf{I}}
\end{aligned}
$$

where $\mathbf{b}$ represents the body force and $\mathbf{f}_{\mathbf{I}}$ represents the mechanical interaction (local exchange of momentum) between two components.

\section{CONSTITUTIVE EQUATIONS}

The plasma is assumed to behave as a linearly viscous fluid:

$$
\mathbf{T}_{1}=\left[-p(1-\phi)+\lambda(1-\phi) \operatorname{tr} \mathbf{D}_{1}\right]+2 \mu(1-\phi) \mathbf{D}_{1}
$$

where $p$ is the fluid pressure, $\mu$ is the viscosity, $\mathbf{D}_{1}$ is the symmetric part of the velocity gradient of the plasma, and $\lambda$ is the second coefficient of viscosity in a compressible fluid.

The stress tensor for the RBCs is assumed to have the structure:

$$
\mathbf{T}_{2}=\beta_{0} \mathbf{I}+\beta_{3} \mathbf{D}_{2}
$$

where $\beta_{0}$ and $\beta_{3}$ are given by Massoudi and Antaki [5]:

$$
\beta_{0}=-p \phi, \quad \beta_{3}=\beta_{30}\left(\phi+\phi^{2}\right)
$$


Shear-thinning effects were incorporated by adopting a shear-dependent viscosity for the RBC phase, introduced by Yeleswarapu et al [6]:

$$
\beta_{30}=\mu_{\infty}+\left(\mu_{0}-\mu_{\infty}\right) \frac{1+\ln (1+\kappa \dot{\gamma})}{1+\kappa \dot{\gamma}}
$$

where $\dot{\gamma}=\left[2 \operatorname{tr}\left(\mathbf{D}_{2}^{2}\right)\right]^{1 / 2}$ is the generalized shear rate, $\mu_{0}$ is the viscosity under zero shear rate, $\mu_{\infty}$ is an asymptotic viscosity for infinite shear rate, and $\kappa$ is a material parameter describing the character of shear thinning.

The mechanical interaction force is assumed to be of the form (Massoudi [7]):

$$
\begin{aligned}
\mathbf{f}_{\mathbf{I}}= & A_{1} \operatorname{grad} \phi+A_{2} F(\phi)\left(\mathbf{v}_{2}-\mathbf{v}_{1}\right) \\
& +A_{3} \phi\left(2 \operatorname{tr} \mathbf{D}_{1}^{2}\right)^{-\frac{1}{4}} \mathbf{D}_{1}\left(\mathbf{v}_{2}-\mathbf{v}_{1}\right)
\end{aligned}
$$

where the terms on the right-hand side of this equation reflect the presence of non-uniform concentration distribution (diffusion), drag, and slip-shear lift, and the coefficients are the same as proposed by Massoudi [7]:

$$
A_{2}=\frac{9}{2} \frac{\mu_{f}}{a^{2}}, A_{3}=\frac{3(6.46)}{4 \pi} \frac{\rho_{f}^{1 / 2} \mu_{f}^{1 / 2}}{a}, \quad F(\phi)=\phi(1+6.55 \phi)
$$

\section{RESULTS}

NUMERICAL SIMULATION OF THE FULLY DEVLOPED FLOW

For numerical simulation, the governing equations are made dimensionless to perform a parametric study for a range of dimensionless numbers. Let us now consider the pressure driven flow of a mixture between two horizontal long flat plates, where $X$ is the direction of the flow, and the plates are located at $\mathrm{Y}=-1$ and $\mathrm{Y}=1$. If the flow is steady and laminar, the velocity profiles and the volume fraction of RBCs can be assumed to have the form:

$$
\mathbf{V}_{1}=\mathrm{V}(\mathrm{Y}) \mathbf{e}_{x}, \quad \mathbf{V}_{2}=\mathrm{U}(\mathrm{Y}) \mathbf{e}_{x}, \quad \phi=\phi(\mathrm{Y})
$$

The equations for balance of mass are automatically satisfied. We use the adherence boundary conditions on both constituents at each plate. A specified value of a for $\phi$ is prescribed at $Y=-1$. We assume

$$
\begin{gathered}
\mathrm{U}(-1)=\mathrm{U}(1)=\mathrm{V}(-1)=\mathrm{V}(1)=0, \\
\phi(-1)=\mathrm{a}
\end{gathered}
$$

Figure 1 shows that the velocity profiles of plasma and $\mathrm{RBC}$, respectively, for a range of dimensionless parameters. In general, the velocity profile of $\mathrm{RBCis}$ observed to be less than that of plasma, but the velocity profile of $\mathrm{RBC}$ approaches that of plasma as lift coefficient (C3) increases. It is found that the velocity profile of $\mathrm{RBC}$ becomes blunter due to increase in $\mathrm{C} 3$. This bluntness also was found as the value of $\kappa$ or the volume fraction of RBCs increases. In figure 2, RBC volume fraction varies nonlinearly from the top wall to the bottom wall due to changes in $\mathrm{C} 3$, is distributed symmetrically at upper and lower plates, and has a peak at the center of the plate due to lift force. This result implies that the volume fraction could be nonlinearly distributed, but asymmetric if gravity would be the same as or greater than an order of the magnitude of lift force.

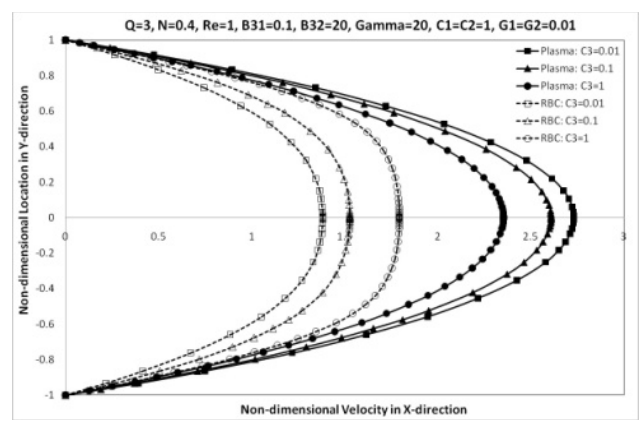

Figure 1: Plots of velocity profiles due to changes in C3.

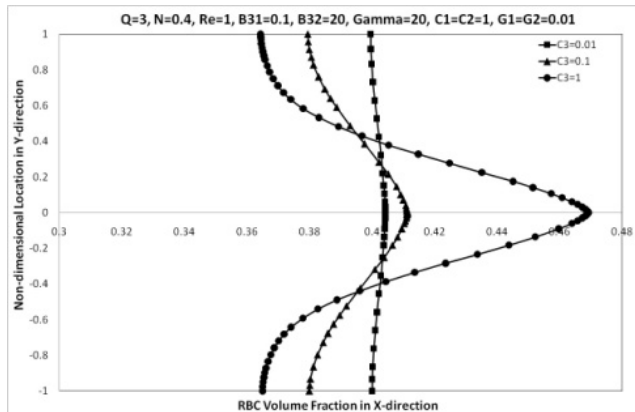

Figure 2: Plots of RBC volume fraction due to changes in $\mathrm{C}$.

\section{ACKNOWLEDGMENTS}

This project was supported by NIH R01 HL089456-01.

\section{REFERENCES}

1. Fung, Y.C., Biomechanics, 3 volumes.

2. Truesdell, C., Rational Thermodynamics. 2nd corr. and enlarged ed. edition ed1984: Springer.

3. Rajagopal, K.R. and L. Tao, Mechanics of Mixtures 1995.

4. Massoudi, M., A mixture theory formulation for hydraulic or pnumatic transport of solid particles. International Journal of Engineering Science, 2010. 48: p. 1440-1461.

5. Massoudi, M. and J.F. Antaki, An Anisotropic Constitutive Equation for the Stress Tensor of Blood Based on Mixture Theory. Mathematical Problems in Engineering, 2008. Article Number: 579172.

6. Yeleswarapu, K.K., Evaluation of continuum models for characterizing the constitutive behavior of blood, 1994, University of Pittsburgh.

7. Massoudi, M., On the importance of material frameindifference and lift forces in multiphase flows. Chemical Engineering Science, 2002. 57(17): p. 36873701. 